\title{
SYNTHESIS OF SOME NEW PRODRUGS OF SULPHONAMIDES AND STUDIES ON THEIR ANTIMICROBIAL AND ANTI-INFLAMMATORY ACTION
}

\author{
M.S.Y. Khan*, A. Husain, S.M. Hasan \& M. Akhter \\ Department of Pharmaceutical Chemistry, Faculty of Pharmacy, Jamia Hamdard (Hamdard \\ University), New Delhi, 110062 , India
}

Various amide-based prodrugs of sulphonamides have been synthesised by condensing appropriate sulphonamide moiety with different $\beta$-aroyl propionic acids. All the compounds have been evaluated for their antimicrobial and anti-inflammatory activities. Their structures were established on the basis of elemental analysis, ${ }^{1} \mathrm{H}$ NMR and Mass spectral data. Some of these compounds were found to have significant activity.

Key words: Sulphonamides, prodrugs, amides, anti-inflammatory, antimicrobial.

\section{Introduction}

The sulphonamides are one of the least expensive chemotherapeutic agents and this factor largely accounts for their greater extent of use in developing countries. They are used in urinary tract infections, meningitis, streptococcal pharyngitis, baciliary dysentery, trachoma, chancroid, malaria, toxoplasmosis, nocardiasis and conjunctivitis ${ }^{1-3}$. Dapsone still remains the drug of 
choice for all forms of leprosy. They are generally taken orally in higher doses which cause nausea, vomiting and epigastric pain ${ }^{4.5}$. $\beta$-Aroyl propionic acids are well known antiinflammatory drugs and some of them are available in the market (fenbufen, bucloxic acid, furobufen etc. $)^{5.6}$, they owe their activity to in-vivo metabolism to the corresponding phenyl acetic acid and have been reported to have comparatively more gastrointestinal side effects as compared to other NSAIDs ${ }^{7-9}$. In view of these facts, it was considered worthwhile to study various amide-based prodrugs of sulphonamides with $\beta$-aroyl propionic acids in order to improve their efficacy and to decrease the side effects. Some times inflammation is caused by microbial infections also and a combination of the antiinflammatory drugs with sulpha drugs will naturally be useful for such conditions. Different $\beta$-aroyl propionic acids were therefore condensed with appropriate sulphonamides and their structures were established on the basis of elemental analysis, ${ }^{1}$ H NMR and Mass spectral data. These compounds were evaluated for their anti-inflammatory and antimicrobial activity.

\section{Results and Discussion}

Fourteen new amide-based prodrugs (7-20) were synthesized by condensing $\beta$-aroyl propionic acid (1-6) with appropriate sulphonamide moiety in molar ratio in dry pyridine in presence of phosphorous oxychloride as condensing agent. The required $\beta$-aroyl propionic acids were prepared by condensing aromatic hydrocarbons or their derivatives with succinic anhydride in presence of anhydrous aluminium chloride following Friedel-Craft's acylation reaction conditions. The physical and analytical data are recorded in Tables-I \& II. 
Table-I: Physical data of the $\beta$-aroyl propionic acids 1- 6<smiles>O=C(O)CCC(=O)[Al]</smiles>

\begin{tabular}{|c|c|c|c|c|}
\hline \multirow[t]{2}{*}{ Compd } & \multirow[t]{2}{*}{ Ar } & \multirow{2}{*}{$\begin{array}{l}\text { Melting } \\
\text { point } \\
\left({ }^{\circ} \mathrm{C}\right) \\
\end{array}$} & \multicolumn{2}{|c|}{ PMR chemical shifts in $\delta$ Values } \\
\hline & & & $\begin{array}{l}\text { Protons of two } \\
\text { methylene groups }\end{array}$ & Ar protons \\
\hline 1 & & 118 & $\begin{array}{l}2.80 \& 3.36(t \\
\left.\text { each, } 2 \mathrm{x}-\mathrm{CH}_{2-}\right)\end{array}$ & $\begin{array}{l}7.46(\mathrm{~m}, 3 \mathrm{H}, \mathrm{H}-3,4,5), 7.84 \\
(\mathrm{~m}, 2 \mathrm{H}, \mathrm{H}-2,6)\end{array}$ \\
\hline 2 & & 180 & $\begin{array}{l}2.82 \& 3.37(t \\
\left.\text { each, } 2 x-C^{-} H_{2^{-}}\right)\end{array}$ & $\begin{array}{l}7.45(\mathrm{~m}, 3 \mathrm{H}, \mathrm{H}-3,4,5), 7.64 \\
(\mathrm{~m}, 2 \mathrm{H}, \mathrm{H}-2,6), 7.7 \& 8.07 \\
\text { (d each, } 2 \mathrm{xA}_{2} \mathrm{~B}_{2}, \quad \mathrm{p}- \\
\text { substituted phenyl) }\end{array}$ \\
\hline 3 & & 172 & $\begin{array}{l}2.8 \& 3.27(t \\
\left.\text { each, } 2 x-\mathrm{CH}_{2-}^{-}\right)\end{array}$ & $\begin{array}{l}7.17(\mathrm{~m}, 3 \mathrm{H}, \mathrm{H}-3,4,5), 7.41 \\
(\mathrm{~m}, 2 \mathrm{H}, \mathrm{H}-2,6), 7.7 \& 7.97 \\
\left(\mathrm{~d} \text { each, } 2 \mathrm{xA}_{2} \mathrm{~B}_{2}, \quad \mathrm{p}-\right. \\
\text { substituted phenyl) }\end{array}$ \\
\hline 4 & & 106 & $\begin{array}{l}2.65 \& 3.26(t \\
\left.\text { each, } 2 x-\mathrm{CH}_{2-}\right)\end{array}$ & $\begin{array}{l}2.37\left(\mathrm{~s}, 3 \mathrm{H},-\mathrm{CH}_{3}\right), 7.27 \& \\
7.85 \text { (d each, } 2 \mathrm{xA}_{2} \mathrm{~B}_{2}, \mathrm{p}- \\
\text { substituted phenyl) }\end{array}$ \\
\hline 5 & & 110 & $\begin{array}{l}2.80 \& 3.30(\mathrm{t} \\
\left.\text { each, } 2 \mathrm{x}-\mathrm{CH}_{2^{-}}\right)\end{array}$ & $\begin{array}{l}1.25\left(\mathrm{t}, 3 \mathrm{H}, \mathrm{CH}_{3} \mathrm{CH}_{2}\right), 2.69 \\
\left(\mathrm{q}, 2 \mathrm{H}, \mathrm{CH}_{3} \mathrm{CH}_{2}\right), 7.27 \& \\
7.90 \text { (d each, } 2 \mathrm{xA}_{2} \mathrm{~B}_{2}, \mathrm{p}- \\
\text { substituted phenyl) }\end{array}$ \\
\hline 6 & & 124 & $\begin{array}{l}2.81 \& 3.38(\mathrm{t} \\
\left.\text { each, } 2 \mathrm{x}-\mathrm{CH}_{2}-\right)\end{array}$ & $\begin{array}{lrr}7.45 \& & 7.92 \quad \text { (d each, } \\
2 \mathrm{xA}_{2} \mathrm{~B}_{2}, & \text { p-substituted } \\
\text { phenyl) } & \end{array}$ \\
\hline
\end{tabular}

$\mathrm{s}=$ singlet $\mathrm{d}=$ doublet $; \mathrm{t}=$ triplet; $\mathrm{q}=$ quatret; $\mathrm{m}=$ multiplet 
Table-II: Physical data of the compounds $7-20$

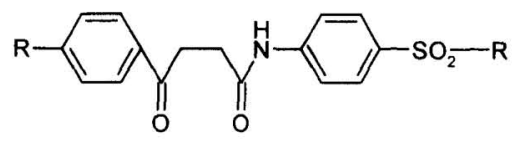

\begin{tabular}{|c|c|c|c|c|c|c|c|}
\hline Compd & $\mathbf{R}$ & $\mathbf{R}^{\prime}$ & $\begin{array}{c}\text { Molecular } \\
\text { Formula; } \\
\text { Mass spectral } \\
\text { data }(\mathbf{m} / \mathbf{z}) \\
\end{array}$ & $\begin{array}{l}\text { M.P. } \\
\left({ }^{\circ} \mathrm{C}\right)\end{array}$ & $\begin{array}{c}\% \\
\text { Yield }\end{array}$ & $\begin{array}{l}\text { Rf - } \\
\text { Value }\end{array}$ & ${ }^{1}$ H NMR ( $\delta$ ppm) \\
\hline 7 & $\mathrm{H}$ & & $\begin{array}{l}\mathrm{C}_{20} \mathrm{H}_{19} \mathrm{O}_{5} \mathrm{~N}_{3} \mathrm{~S} \\
413\left(\mathrm{M}^{+}\right), 316 \\
161,105,77\end{array}$ & $\begin{array}{c}188- \\
90\end{array}$ & 70 & 0.74 & 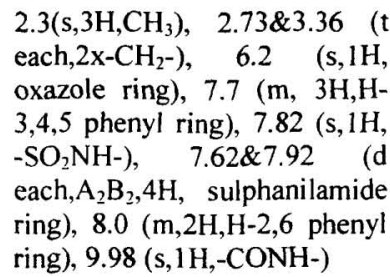 \\
\hline 8 & $\mathrm{H}$ & & $\begin{array}{c}\mathrm{C}_{32} \mathrm{H}_{28} \mathrm{O}_{6} \mathrm{~N}_{2} \mathrm{~S} \\
\text { Not recorded }\end{array}$ & 204 & 42 & 0.6 & $\begin{array}{l}2.9 \& 3.6\left(\mathrm{t} \text { each, } 2 \mathrm{x}-\mathrm{CH}_{2}-\mathrm{CH}_{2}-\right) \\
7.5(\mathrm{~m}, 6 \mathrm{H}, 2 \mathrm{xH}-3,4,52 \mathrm{x} \text { phenyl } \\
\text { ring), } 7.69(\mathrm{~d}, 4 \mathrm{H}, 2 \mathrm{x} \text { hydrogens } \\
\text { ortho to the amino group), } 7.98 \\
(\mathrm{~d}, 4 \mathrm{H}, 2 \mathrm{x} \text { hydrogens ortho to } \\
\mathrm{SO}_{2} \text { group), } 7.88(\mathrm{~m}, 4 \mathrm{H}, 2 \mathrm{xH}- \\
2,62 \mathrm{x} \text { - phenyl ring) }\end{array}$ \\
\hline 9 & $\mathrm{H}$ & $-\mathrm{NH}_{2}$ & $\begin{array}{c}\mathrm{C}_{16} \mathrm{H}_{16} \mathrm{O}_{4} \mathrm{~N}_{2} \mathrm{~S} \\
322\left(\mathrm{M}^{+}\right), 161, \\
105,77\end{array}$ & 194 & 50 & 0.68 & $\begin{array}{l}\left.2.76 \& 3.4 \text { (t each, } 2 \mathrm{x}-\mathrm{CH}_{2}-\right), \\
\left.6.74 \text { (s,2H,-SO } \mathrm{NH}_{2}\right), \quad 7.27 \\
(\mathrm{~m}, 3 \mathrm{H}, \mathrm{H}-3,4,5 \text { phenyl ring), } \\
8.0(\mathrm{~m}, 2 \mathrm{H}, \mathrm{H}-2,6 \text { phenyl ring), } \\
7.7 \& 7.92 \text { (d each, } \mathrm{A}_{2} \mathrm{~B}_{2}, 4 \mathrm{H}, \\
\text { sulphanilamide ring), } 10.08 \\
(\mathrm{~s}, 1 \mathrm{H},-\mathrm{CONH}-)\end{array}$ \\
\hline 10 & $\mathrm{H}$ & & $\begin{array}{c}\mathrm{C}_{19} \mathrm{H}_{16} \mathrm{O}_{4} \mathrm{~N}_{4} \mathrm{~S} \\
396\left(\mathrm{M}^{+}\right), 234, \\
161,105\end{array}$ & $\begin{array}{c}144- \\
46\end{array}$ & 46 & 0.71 & 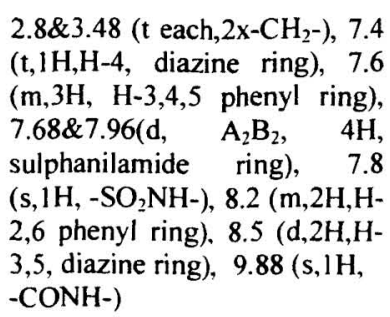 \\
\hline
\end{tabular}


11<smiles>Cc1ccccc1</smiles><smiles>CNc1cc(O)on1</smiles>

$\mathrm{C}_{26} \mathrm{H}_{23} \mathrm{O}_{5} \mathrm{~N}_{3} \mathrm{~S} \quad 212$

$488\left(\mathrm{M}^{+}\right), 470$

$236,180,152$

12<smiles>Cc1ccccc1</smiles>

$-\mathrm{NH}_{2}$

$\mathrm{C}_{26} \mathrm{H}_{23} \mathrm{O}_{5} \mathrm{~N}_{3} \mathrm{~S} 198$

60

$408\left(M^{+}\right), 390$,

$236,152,77$

13<smiles>[O-]c1ccccc1</smiles><smiles>CNc1cc(C)on1</smiles>

$\mathrm{C}_{26} \mathrm{H}_{23} \mathrm{O}_{6} \mathrm{~N}_{3} \mathrm{~S} \quad 208$

56

14<smiles>c1ccc([OH+]c2ncccn2)cc1</smiles>

$\mathrm{C}_{25} \mathrm{H}_{20} \mathrm{O}_{5} \mathrm{~N}_{4} \mathrm{~S} \quad 168 \quad 51$

$488\left(\mathrm{M}^{+}\right), 424$,

$253,234,197$

15<smiles>CNc1ncccn1</smiles>

$\mathrm{C}_{20} \mathrm{H}_{18} \mathrm{O}_{4} \mathrm{~N}_{4} \mathrm{~S}$
$206-$
$424\left(\mathrm{M}^{+}\right), 360$

$342,175,119$

91,77
$0.762 .3\left(\mathrm{~s}, 3 \mathrm{H}, \mathrm{CH}_{3}\right), \quad 2.86 \& 3.44 \quad(\mathrm{t}$ each, $\left.2 \mathrm{x}-\mathrm{CH}_{2}-\right), 6.06(\mathrm{~s}, 1 \mathrm{H}$, oxazole ring), $7.7 \& 7.78$ (d each, $A_{2} B_{2}, 4 \mathrm{H}, \quad$ p-substituted phenyl ring), $7.8 \& 8.07$ (d each, $\mathrm{A}_{2} \mathrm{~B}_{2}, \quad 4 \mathrm{H}, \quad$ sulphanilamide ring), 7.4-7.62 (m,5H, phenyl ring hydrogens $), 7.82(\mathrm{~s}, 1 \mathrm{H}$,

$\left.-\mathrm{SO}_{2} \mathrm{NH}-\right), 9.97(\mathrm{~s}, 1 \mathrm{H},-\mathrm{CONH}-)$

$0.782 .82 \& 3.34$ ( $\mathrm{t}$ each, $2 \mathrm{x}-\mathrm{CH}_{2}$-), $7.26 \& 7.7$ (d each, $\mathrm{A}_{2} \mathrm{~B}_{2}, 4 \mathrm{H}$, psubstituted phenyl ring), $7.77 \& 7.94$ (d each, $A_{2} B_{2}, 4 H$, sulphanilamide ring), 7.07-7.2 ( $\mathrm{m}, 5 \mathrm{H}$, phenyl ring hydrogens), 7.62 (s, $2 \mathrm{H}, \quad-\mathrm{SO}_{2} \mathrm{NH}_{2}$ ), 10.04 (s, $1 \mathrm{H},-\mathrm{CONH}-)$

$0.622 .3\left(\mathrm{~s}, 3 \mathrm{H}, \mathrm{CH}_{3}\right), \quad 2.79 \& 3.34 \quad(\mathrm{t}$ each, $\left.2 \mathrm{x}-\mathrm{CH}_{2}-\right), 6.04(\mathrm{~s}, 1 \mathrm{H}$, oxazole ring), 7.1\&7.7 (d each, $\mathrm{A}_{2} \mathrm{~B}_{2}, 4 \mathrm{H}$, p-substituted phenyl ring), 7.77\&7.94 (d each $A_{2} B_{2}, 4 H$, sulphanilamide ring) $7.07-7.2(\mathrm{~m}, 5 \mathrm{H}$, phenyl ring hydrogens $), 7.6(\mathrm{~s}, 1 \mathrm{H}$, $\left.-\mathrm{SO}_{2} \mathrm{NH}-\right), 10.11(\mathrm{~s}, 1 \mathrm{H}$, $-\mathrm{CONH}-)$

2.8\&3.42(t each, $2 \mathrm{x}-\mathrm{CH}_{2}-$ ), 6.97 \&7.6 (d each, $\mathrm{A}_{2} \mathrm{~B}_{2}, 4 \mathrm{H}$, psubstituted phenyl ring), 7.94 $\& 7.97$ (d each, $A_{2} B_{2}, 4 H$, sulphanilamide ring), 6.8-7.2 ( $\mathrm{m}, 5 \mathrm{H}$, phenyl ring hydrogens), 7.4 ( $\mathrm{t}, 1 \mathrm{H}, \mathrm{H}-4$, diazine ring), 8.5 (d,2H,H-3,5,diazine ring), 7.8 (s, $\left.1 \mathrm{H}, \mathrm{SO}_{2} \mathrm{NH}-\right), 10.12(\mathrm{~s}, 1 \mathrm{H}$, $-\mathrm{CONH}-)$

$\begin{array}{lllll}0.40 & 2.41 \quad\left(\mathrm{~s}, 3 \mathrm{H}, \mathrm{CH}_{3}\right) & 2.8 \& 3.34 \quad(\mathrm{t}\end{array}$ each, $\left.2 \mathrm{x}-\mathrm{CH}_{2}-\right), 6.9(\mathrm{t}, \mathrm{lH}, \mathrm{H}-$ 4, diazine ring), 7.27 (m,2H,H3,5 , diazine ring), $7.62(\mathrm{~s}, 1 \mathrm{H}$, $-\mathrm{SO}_{2} \mathrm{NH}-$ ), $7.75 \& 8.4$ (d each, $\mathrm{A}_{2} \mathrm{~B}_{2}, 4 \mathrm{H}$, p-substituted phenyl ring), $7.89 \& 7.9$ (d each, $\mathrm{A}_{2} \mathrm{~B}_{2}$, $4 \mathrm{H}$, p-substituted toluene ring), $10.16(\mathrm{~s}, 1 \mathrm{H},-\mathrm{CONH}-)$ 


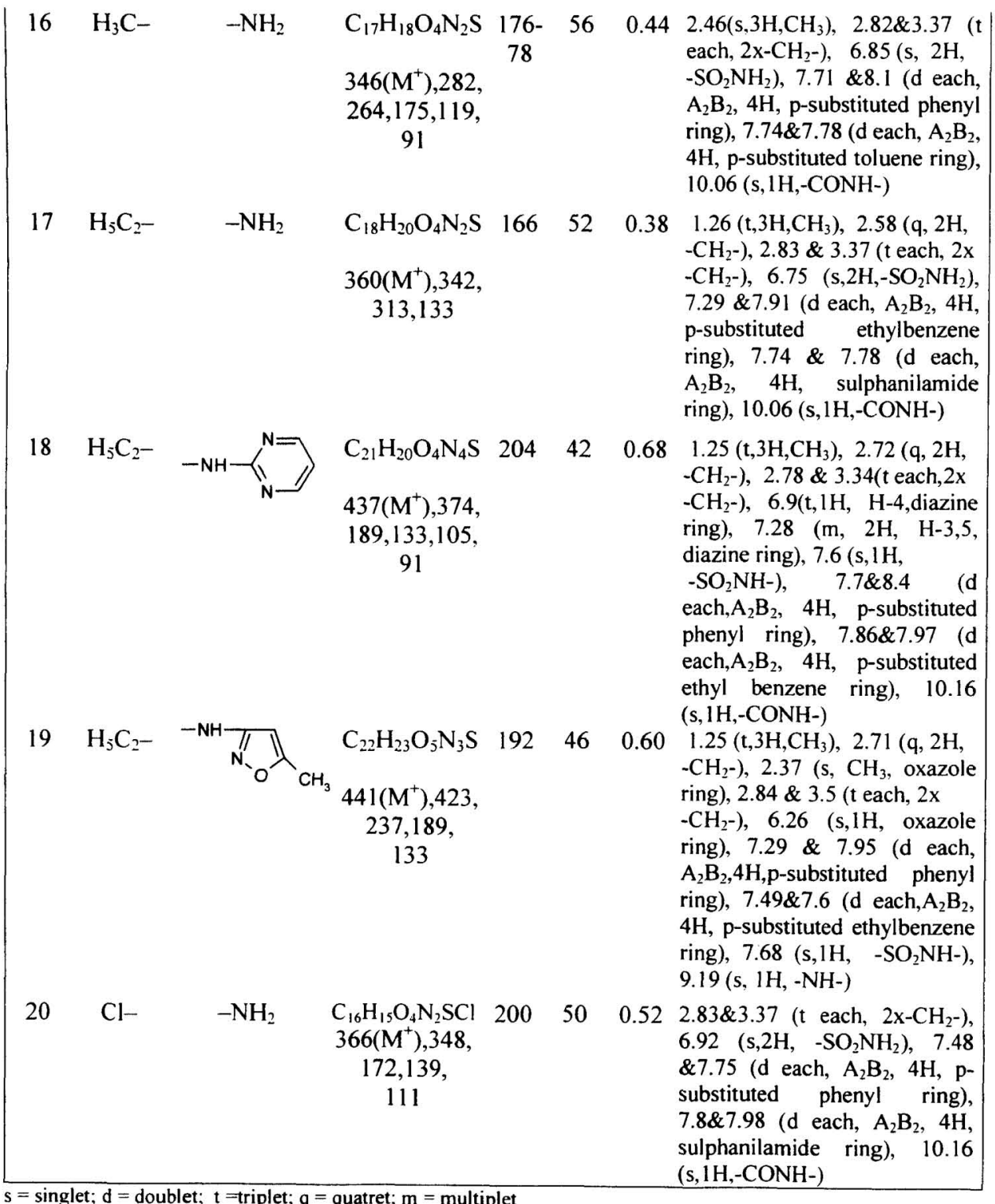


Anti-inflammatory activity

Carrageenan induced rat paw oedema method was employed for evaluating the antiinflammatory activity of the compounds at a dose level of $20 \mathrm{mg} / \mathrm{kg} \mathrm{b.w}$. in albino rats (weighing 100-120 gm) using indomethacin as a standard drug for comparision. The rat paw oedema was produced by the method of Winter et $a l^{10}$. The percentage inhibition of inflammation was calculated by applying Newbould formula ${ }^{11}$. In this test, the most active compounds were 13 and 14 which showed $58.06 \%$ and $54.83 \%$ inhibition respectively and their activity was comparable with the standard drug indomethacin (61.30\%) at a dose of $20 \mathrm{mg} / \mathrm{kg}$ body weight. Results are presented in Table-III. It is significant that none of these compounds showed ulcerogenic activity, which is a common feature with NSAIDs. The ulcerogenic activity was carried out by the reported method ${ }^{12}$.

\section{Antibacterial activity}

The bacterial strains gram positive (Staphylococcus aureus) and gram negative (Esherichia coli) were used. The test was carried out according to the turbidity method ${ }^{13}$. A solution of the compounds was prepared in dimethylformamide (DMF) and a series of doubling dilutions prepared with sterile pipettes. To each of a series of sterile stoppered test tubes a standard volume of nutrient broth medium was added. A control tube containing no antimicrobial agent was included. The inoculum consisting of an overnight broth culture of microorganisms was added to separate tubes. The tubes were incubated at $37^{\circ}$ for 24 hours and examined for turbidity. The tubes with highest dilution showing no turbidity was the MIC. Compound 9 
showed excellent activity against Staphylococcus aureus (MIC- $5 \mu \mathrm{g} / \mathrm{ml}$ ) while compound 20 was highly active against Escherichia coli (MIC-10 $\mu \mathrm{g} / \mathrm{ml})$. Results are presented in Table-III.

Table-III: Biological activity

\begin{tabular}{|c|c|c|c|c|c|}
\hline \multirow[t]{2}{*}{ Compd } & \multicolumn{2}{|c|}{$\begin{array}{l}\text { Antibacterial Activity } \\
\text { (Minimum Inhibitory } \\
\text { Concentration*) }\end{array}$} & \multicolumn{3}{|c|}{$\begin{array}{l}\text { Anti-inflammatory Activity } \\
\text { (\% Inhibition in Rat paw oedema) }\end{array}$} \\
\hline & S. aureus & E. coli & $\begin{array}{l}\text { Normal paw } \\
\text { Volume }(x)\end{array}$ & $\begin{array}{l}\text { Paw oedema } \\
5 \mathrm{hr} \text { after } \\
\text { carragenan (a) }\end{array}$ & $\begin{array}{c}\% \text { inhibition } \\
(1-\mathrm{a}-\mathrm{x} / \mathrm{b}-\mathrm{y}) 100 \\
\text { of oedema }\end{array}$ \\
\hline 6 & $>100$ & $>100$ & $0.65 \pm 0.03$ & $0.92 \pm 0.02$ & 12.90 \\
\hline 7 & - & - & $0.73 \pm 0.03$ & $0.95 \pm 0.04$ & 29.03 \\
\hline 8 & $>100$ & & $0.67 \pm 0.03$ & $0.93 \pm 0.04$ & 16.12 \\
\hline 9 & 5 & 50 & $0.65 \pm 0.03$ & $0.90 \pm 0.02$ & 19.35 \\
\hline 10 & $>100$ & - & $0.70 \pm 0.03$ & $0.93 \pm 0.03$ & 25.80 \\
\hline 11 & $>100$ & $>100$ & $0.73 \pm 0.03$ & $0.95 \pm 0.04$ & 29.03 \\
\hline 12 & $>100$ & - & $0.71 \pm 0.04$ & $0.96 \pm 0.05$ & 19.35 \\
\hline 13 & - & - & $0.68 \pm 0.03$ & $0.81 \pm 0.03$ & 58.06 \\
\hline 14 & - & - & $0.72 \pm 0.02$ & $0.86 \pm 0.03$ & 54.83 \\
\hline 15 & $>100$ & - & $0.71 \pm 0.02$ & $0.88 \pm 0.03$ & 45.16 \\
\hline 16 & 25 & $>100$ & $0.72 \pm 0.02$ & $0.97 \pm 0.03$ & 19.35 \\
\hline 17 & 50 & $>100$ & $0.69 \pm 0.03$ & $0.91 \pm 0.03$ & 29.03 \\
\hline 18 & $>100$ & $>100$ & $0.68 \pm 0.02$ & $0.88 \pm 0.03$ & 35.48 \\
\hline 19 & - & - & $0.68 \pm 0.03$ & $0.92 \pm 0.03$ & 22.58 \\
\hline 20 & 25 & 10 & $0.66 \pm 0.02$ & $0.89 \pm 0.04$ & 25.80 \\
\hline \multicolumn{3}{|c|}{ Indomethacin } & $0.73 \pm 0.03$ & $0.85 \pm 0.03$ & 61.30 \\
\hline \multicolumn{2}{|c|}{ Control } & & $0.68 \pm 0.02(y)$ & $0.99 \pm 0.03(\mathrm{~b})$ & \\
\hline
\end{tabular}

\section{EXPERIMENTAL}

Melting points were taken in open capillary tubes and are uncorrected. Analytical data of $\mathrm{C}, \mathrm{H}$, $\mathrm{O}, \mathrm{N}$, and $\mathrm{S}$ were within $\pm 0.4 \%$ of the theoretical values. ${ }^{1} \mathrm{H}$ NMR spectra were recorded on Varian E-360 MHz or Bruker spectropsin DPX-300MHz with tetramethylsilane as internal 
standard in solvent $\mathrm{CDCl}_{3}$. Mass spectra of the compounds were recorded on a JEOL-DX 303 instrument. TLC were carried out using silica gel (Merck No. 5554). Dry solvents were used throughout.

\section{ß-Aroyl propionic acids 1-6}

Succinic anhydride $(0.1$ mole) was condensed in presence of anhydrous aluminium chloride (0.1125 mole) with appropriate aromatic compounds in equimolar ratio. The reactions were carried out in dry solvents (nitrobenzene or 1,1,2,2-tetrachloroethane). In case of aromatic compounds which were liquid in nature, no solvent was used and they were taken in excess. The reaction mixture was refluxed for two hours and excess solvent was removed by steam distillation. It was purified by dissolving in sodium hydroxide solution. filtering, followed by addition of hydrochloric acid. A solid mass so obtained was filtered, washed with cold water. dried and crystallized from methanol to give 1-6 (Table-1).

\section{Amides 7-20}

Amides were synthesized by dissolving $\beta$-aroyl propionic acid and sulphonamide (equimolar: 3 mmole) in minimum quantity of dry pyridine separately. The two solutions were then mixed together and stirred magnetically followed by the addition of phosphorous oxychloride $(0.9 \mathrm{ml})$ dropwise while maintaining the temperature below $5^{\circ}$. The contents were stirred for another half-hour and left overnight. The reaction mixture was then poured into ice cold water and a solid mass, which separated out, was filtered, washed, dried and crystallized from ethanol to give 7-20 (Table-II). 


\section{References}

1 Northey E. H. (1948), The sulfonamides and allied compounds, American Chemical Society Monograph Sereis, Reinhold, NewYork.

2 Sabin A. B., Warren J. (1941), J. Bacteriol. 41 (M 50): 80

3 Forster W. G., McGlbeny J. R. (1944), Am. J. Ophthalmol. 27C: 1107

4 Schwartz W. B. (1949), New Engl. J. Med. 240

5 Anand N. (1979), Wolf M. E. (ed.), Burger`s Medicinal Chemistry, ed.4, WileyInterscience-NewYork, p. 34.

6 Husain A. (2000), Ph.D. Thesis, Jamia Hamdard (Hamdard University), New Delhi, India.

7 Testa B., Jenner P. (1976), Drug Metabolism, Chemical and Biochemical aspect, Marcel Dekker Inc-New York, p. 138.

8 Kohler C., Tolman E., Wooding W. Ellenbogen L. (1980), Arzneim-Forsch 30(4A): 702

9 Sloboda A. E., Tolman E. L., Osterberg A. C., Panagides J. (1980), Arzneim-Forsch 30(4A), 716.

10 Winter C. A., Risley E. A. Nuss G. N. (1962), Proc. Soc. Exp. Biol. 111: 544.

11 Newbould B. B. (1963), Brit. J. Pharmacol. 21: 157.

12 Wilhemi G., Menass-Gdynia R. (1972), Pharmacology 8: 321.

13 Cruickshank R., Dugid J. P., Marmion D. P. Swain R. H. A. (1975), Medical Microbiology Vol. 2. Churchill-Livingstone - Edinburge - London, p. 2. 